Classification

Physics Abstracts

$61.40 \mathrm{~K}-36.20$

\title{
Long polymers in good solvent : $\varepsilon$-expansion of the ratio of the radius of gyration to the end to end distance
}

\author{
M. Benhamou and G. Mahoux \\ Service de Physique Théorique, CEN-Saclay, 91191 Gif-sur-Yvette Cedex, France
}

(Reçu le 8 avril 1985, accepté sous forme définitive le 6 juin 1985)

\begin{abstract}
Résumé. - Le rapport du rayon de giration à la distance carrée moyenne bout à bout pour une longue chaîne isolée est donné au second ordre en $\varepsilon=4-d$, où $d$ est la dimension de l'espace. Notre méthode de calcul est basée sur le concept de renormalisation directe introduit par J. des Cloizeaux. A trois dimensions $(\varepsilon=1)$, notre résultat peut être comparé au résultat numérique obtenu par C. Domb et F. F. Hioe.
\end{abstract}

\begin{abstract}
The ratio of the radius of gyration to the mean square end to end distance of an isolated long chain is given to second order in $\varepsilon=4-d$, where $d$ is the space dimension. Our method of calculation is based on the direct renormalization idea introduced by J. des Cloizeaux. In dimension three $(\varepsilon=1)$, our result can be compared with the result of computer experiments made by C. Domb and F. F. Hioe.
\end{abstract}

\section{Introduction.}

The ratio of the radius of gyration to the mean square end to end distance of an isolated chain is a finite quantity having a universal character in the long chain limit. Our aim here, is to calculate this ratio to second order in $\varepsilon=4-d$. The strategy consists in making the calculation near dimension four, and extending the result to dimension three $(\varepsilon=1)$. The model is the continuous one $[1,2,3]$ in which a polymer in a good solvent is represented by a Brownian chain with local interaction. In the framework of this model, a chain is considered as a continuous curve in a continuous space of dimension $d$. A point on the chain is given by a vector $\mathbf{r}(s)(0<s<S)$, where the parameter $s$ marks the position of this point on the chain (in terms of discrete chain $s$ is proportional to the number of links), and the quantity $S$ is the area of the Brownian chain.

The mean square end to end distance of an isolated chain is defined by

$$
R^{2}=\left\langle[\mathbf{r}(S)-\mathbf{r}(0)]^{2}\right\rangle
$$

and the radius of gyration by :

$$
R_{G}^{2}=\frac{1}{2 S^{2}} \int_{0}^{S} \mathrm{~d} s_{1} \int_{0}^{S} \mathrm{~d} s_{2}\left\langle\left[\mathbf{r}\left(s_{2}\right)-\mathbf{r}\left(s_{1}\right)\right]^{2}\right\rangle
$$




\section{Calculations and result.}

The mean values which appear in the above formulae can be expressed in terms of partition functions as follows :

$$
\begin{gathered}
\left\langle[\mathbf{r}(S)-\mathbf{r}(0)]^{2}\right\rangle=\left.\frac{-2 d}{Z(S, b)} \frac{\partial}{\partial k^{2}} Z(\mathbf{k},-\mathbf{k} ; S, b)\right|_{\mathbf{k}=\mathbf{0}}, \\
\left\langle\left[\mathbf{r}\left(s_{1}\right)-\mathbf{r}\left(s_{2}\right)\right]^{2}\right\rangle=-\left.\frac{2 d}{Z(S, b)} \frac{\partial}{\partial k^{2}} Z\left(\mathbf{k},-\mathbf{k} ; s_{1}, s_{2} ; S, b\right)\right|_{\mathbf{k}=\mathbf{0}} .
\end{gathered}
$$

Here, $Z(S, b)$ is the partition function of one polymer; $Z(\mathbf{k},-\mathbf{k} ; S, b)$ is the partition function of one polymer with two insertions of wave vectors $\mathbf{k}$ and $-\mathbf{k}$ respectively at the origin and extremity of the chain $Z\left(\mathbf{k},-\mathbf{k} ; s_{1}, s_{2} ; S, b\right)$ has the same definition as $Z(\mathbf{k},-\mathbf{k} ; S, b)$, except that the wave vectors $\mathbf{k}$ and $-\mathbf{k}$ are injected at two points of coordinates $s_{1}$ and $s_{2}$ along the chain.

The three partition functions $Z(S, b), Z(\mathbf{k},-\mathbf{k} ; S, b)$ and $Z\left(\mathbf{k},-\mathbf{k} ; s_{1}, s_{2} ; S, b\right)$ are expanded with respect to the coupling constant $b$. The diagrams that contribute up to second order in $b$ to the two first partition functions are exhibited in figure 1 . To the same order in $b$, the diagrams that contribute to the third partition function are those of figure 1 modified by two insertions in all possible positions.

Actually, we need the perturbation expansion of $Z(S, b)$ only to first order. We write :

$$
Z(S, b)=1+Z_{1}(z)+\mathbf{O}\left(z^{2}\right),
$$

where $z$ is the dimensionless parameter

$$
z=(2 \pi)^{-d / 2} b S^{2-d / 2} .
$$

Similarly, $R^{2}$ and $R_{\mathrm{G}}^{2}$ can be written :

$$
\begin{aligned}
& R^{2}=d S\left[1-I_{1}-I_{2}-I_{3}-I_{4}+Z_{1} I_{1}+\mathbf{O}\left(z^{3}\right)\right], \\
& R_{\mathrm{G}}^{2}=d S\left[\frac{1}{6}-J_{1}-J_{2}-J_{3}-J_{4}+Z_{1} J_{1}+\mathbf{O}\left(z^{3}\right)\right],
\end{aligned}
$$

where the 8 quantities $I_{1}, \ldots, I_{4}, J_{1}, \ldots, J_{4}$, functions of the only parameter $z$, have an index running from 1 to 4 , referring to one of the 4 diagrams of figure 1 .

One knows that at any (non necessarily integer) dimension $d$ less than $4, R^{2}$ and $R_{\mathrm{G}}^{2}$ are divergenceless quantities. Accordingly, we need not introduce any cut-off in our calculation as long as
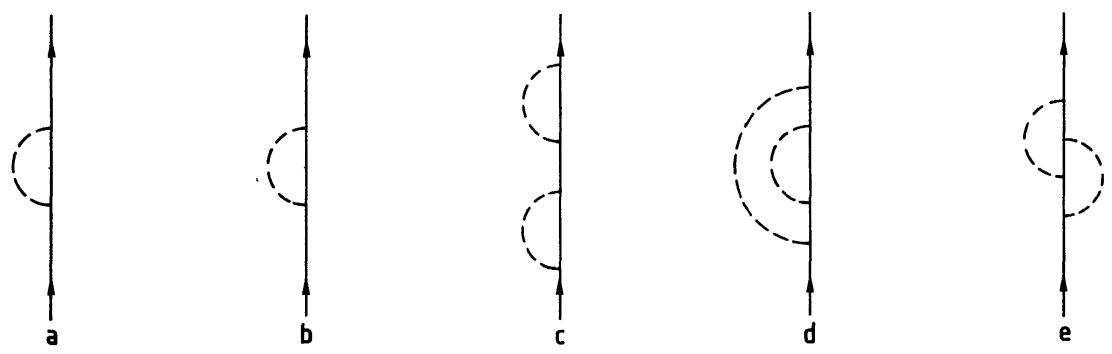

Fig. 1. - Diagrams of first and second order contributing to $Z(S, b)$ and $Z(\mathbf{k},-\mathbf{k} ; S, b)$. 
$d<4$. However, $Z_{1}, I_{2}, I_{3}, J_{2}$ and $J_{3}$ exhibit simple poles at $d=2$. We have checked that the particular combinations $I_{2}+I_{3}-Z_{1} I_{1}$ and $J_{2}+J_{3}-Z_{1} J_{1}$ that enter the expressions of $R^{2}$ and $R_{\mathrm{G}}^{2}$ are pole free, as expected.

The ratio $R_{\mathrm{G}}^{2} / R^{2}$ now reads

$$
\frac{R_{\mathrm{G}}^{2}}{R^{2}}=\frac{1}{6}\left\{1+\sum_{D=1}^{4}\left(I_{D}-6 J_{D}\right)+\left(I_{1}-Z_{1}\right)\left(I_{1}-6 J_{1}\right)+\mathbf{O}\left(z^{3}\right)\right\} .
$$

The 3 first order quantities $Z_{1}, I_{1}$ and $J_{1}$ have a simple pole at $d=4$, whereas the 6 second order ones, $I_{2}, \ldots, J_{4}$, have double poles. We have observed that the combinations $I_{D}-6 J_{D}(D=2,3,4)$ have only simple poles at $d=4$, and that $I_{1}-6 J_{1}$ is pole free. For the various quantities appearing in equation (1), we have obtained the following $\varepsilon(=4-d)$ expansions :

$$
\begin{aligned}
& I_{1}-Z_{1}=-\frac{4 z}{\varepsilon}+\mathbf{O}(\varepsilon), \\
& I_{1}-6 J_{1}=-\frac{z}{12}\left[1-\frac{5 \varepsilon}{12}+\mathbf{O}\left(\varepsilon^{2}\right)\right] \\
& I_{2}-6 J_{2}=z^{2}\left[\frac{1}{3 \varepsilon}-\frac{17}{18}+\mathbf{O}(\varepsilon)\right], \\
& I_{3}-6 J_{3}=z^{2}\left[-\frac{1}{2 \varepsilon}+\frac{11}{12}+\mathbf{O}(\varepsilon)\right], \\
& I_{4}-6 J_{4}=z^{2}\left[\frac{1}{2 \varepsilon}-\frac{1}{24}-\frac{5 \xi}{6}+\mathbf{O}(\varepsilon)\right],
\end{aligned}
$$

where $\xi$ is defined by

$$
\begin{aligned}
\xi & =\frac{2 \pi}{\sqrt{3}} \log \frac{4}{3}-6 \int_{0}^{1} \mathrm{~d} u \frac{\log \left(1-u^{2}\right)}{1+3 u^{2}} \\
& =2.343907235 \ldots
\end{aligned}
$$

This gives for the ratio $R_{\mathrm{G}}^{2} / R^{2}$ the following double expansion :

$$
\frac{R_{\mathrm{G}}^{2}}{R^{2}}=\frac{1}{6}\left[1+A_{1} z+A_{2} z^{2}+\mathbf{O}\left(z^{3}\right)\right]
$$

with

$$
\begin{aligned}
& A_{1}=-\frac{1}{12}\left[1-\frac{5}{12} \varepsilon+\mathbf{O}\left(\varepsilon^{2}\right)\right] \\
& A_{2}=\frac{2}{3 \varepsilon}-\frac{5}{6}\left(\xi+\frac{1}{4}\right)+\mathbf{O}(\varepsilon) .
\end{aligned}
$$

We note that $A_{2}$ is singular at $\varepsilon=0$.

Now, the renormalization strategy consists in dropping the variable $z$ to the benefit of a more physical quantity $g$, which is (essentially) the second virial coefficient. This can be done by using the expansion of $z$ in terms of $g[3]$ :

$$
z=g+\frac{g^{2}}{\varepsilon}[8-(2+4 \operatorname{Ln} 2) \varepsilon]+\mathbf{O}\left(g^{3}\right)
$$


This expansion is also singular at $\varepsilon=0$. Inserting it in equations (2) and (3), we obtain :

$$
\frac{R_{\mathrm{G}}^{2}}{R^{2}}=\frac{1}{6}\left[1-\frac{1}{12}\left(1-\frac{5 \varepsilon}{12}\right) g+\frac{1}{6}\left(\frac{17}{12}+2 \operatorname{Ln} 2-5 \xi\right) g^{2}+\mathbf{O}\left(g^{3}\right)\right]
$$

and we observe that the singularity at $\varepsilon=0$ has now disappeared.

This is in agreement with the fact that relations between physical quantities should remain meaningful up to dimension 4.

Finally, when $z \rightarrow \infty$ (Kuhnian chain limit), $g \rightarrow g^{*}$, with [3]

$$
g^{*}=\frac{\varepsilon}{8}+\frac{\varepsilon^{2}}{16}\left(\frac{25}{16}+\operatorname{Ln} 2\right)+\mathbf{O}\left(\varepsilon^{3}\right)
$$

In this limit the ratio $R_{\mathrm{G}}^{2} / R^{2}$ becomes

$$
\frac{R_{\mathrm{G}}^{2}}{R^{2}}=\frac{1}{6}\left[1-\frac{\varepsilon}{96}-0.0306 \varepsilon^{2}+\mathbf{O}\left(\varepsilon^{3}\right)\right] \text {. }
$$

\section{Comments.}

The two first terms of the $\varepsilon$-expansion of the ratio $R_{\mathrm{G}}^{2} / R^{2}$ (Eq. (4)) have already been found by J. Des Cloizeaux [3] and by T. A. Witten et al. [4] who used the $n=0$ limit of an $n$-component $\phi^{4}$ field theory. For $d=3(\varepsilon=1)$ the $\varepsilon^{2}$ correction to $R_{\mathrm{G}}^{2} / R^{2}$ gives a value of 0.159 which is to be compared to 0.165 in first order. Notice that the value of computer experiments obtained by C. Domb et al. [5] is equal to 0.155 . The $\varepsilon^{2}$ correction provides an improvement of the first order calculation, since it yields a result closer to the value of the numerical simulation. We represent on figure 2 , with respect to $\varepsilon$, the variation of $6 R_{\mathrm{G}}^{2} / R^{2}$.

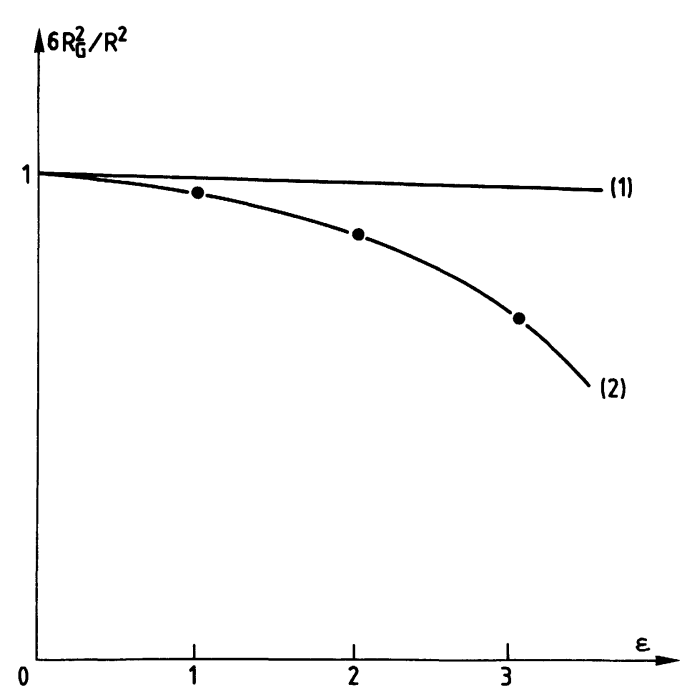

Fig. 2. - (1) The variation of $6 R_{\mathrm{G}}^{2} / R^{2}$ at first order with respect to $\varepsilon$. (2) The variation of $6 R_{\mathrm{G}}^{2} / R^{2}$ at second order with respect to $\varepsilon$. 
Concerning this subject, we recall that Peterlin [6], Benoit [7] and Ptitsyn [8] using a simple assumption (the swelling law is uniform on the chain) have derived an approximate expression of $R_{\mathrm{G}}^{2} / R^{2}$ :

$$
\begin{aligned}
\frac{R_{\mathrm{G}}^{2}}{R^{2}} & =\int_{0}^{1} \mathrm{~d} t(1-t) t^{2 v} \\
& =\frac{1}{(2 v+1)(2 v+2)} .
\end{aligned}
$$

On the other hand the exponent $v$ is given by [3] :

$$
v=\frac{1}{2}\left[1+\frac{\varepsilon}{8}+\frac{15}{256} \varepsilon^{2}+\mathbf{O}\left(\varepsilon^{3}\right)\right] .
$$

This leads to the result :

$$
\frac{R_{\mathrm{G}}^{2}}{R^{2}}=\frac{1}{6}\left[1-\frac{5}{48} \varepsilon-\frac{187}{4608} \varepsilon^{2}+\mathbf{O}\left(\varepsilon^{3}\right)\right]
$$

which is really different from equation (4) and gives the value of 0.142 at $d=3(\varepsilon=1)$. Of course, we cannot believe this result since it relies on an unrealistic assumption.

\section{Acknowledgments.}

It is a pleasure for us to thank J. des Cloizeaux who suggested the subject and also M. Daoud and B. Duplantier for helpful discussions.

\section{References}

[1] Edwards, S. F., Proc. Phys. Soc. 85 (1965) 613.

[2] Yamakawa, H., Modern Theory of Polymer Solutions (Harper and Row) 1971.

[3] Des Cloizeaux, J., J. Physique 42 (1981) 635.

[4] Witten, T. A. et Schäfer, L., J. Phys. A 11 (1978) 1843.

[5] Domb, C. et Hioe, F. T., J. Chem. Phys. 51 (1969) 223.

[6] Peterlin, A. J., J. Chem. Phys. 23 (1955) 2464.

[7] Benoit, H., C.R. Acad. Sci. Paris 245 (1957) 2244.

[8] Ptitsyn, O. B., Zh. Fiz. Khim 31 (1957) 1091. 